\title{
Approximating the inspiral of test bodies into Kerr black holes
}

\author{
Kostas Glampedakis \\ Department of Physics and Astronomy, Cardiff University, P.O. Box 913, Cardiff CF24 3YB, United Kingdom \\ Scott A. Hughes \\ Institute for Theoretical Physics, University of California, Santa Barbara, California 93103 \\ Daniel Kennefick \\ Theoretical Astrophysics, California Institute of Technology, Pasadena, California 91125
}

(Received 8 May 2002; published 12 September 2002)

\begin{abstract}
We present a new approximate method for constructing gravitational radiation driven inspirals of test bodies orbiting Kerr black holes. Such orbits can be fully described by a semilatus rectum $p$, an eccentricity $e$, and an inclination angle $\iota$, or, by an energy $E$, an angular momentum component $L_{z}$, and a third constant $Q$. Our scheme uses expressions that are exact (within an adiabatic approximation) for the rates of change $(\dot{p}, \dot{e}, i)$ as linear combinations of the fluxes $\left(\dot{E}, \dot{L}_{z}, \dot{Q}\right)$, but uses quadrupole-order formulas for these fluxes. This scheme thus encodes the exact orbital dynamics, augmenting it with an approximate radiation reaction. Comparing inspiral trajectories, we find that this approximation agrees well with numerical results for the special cases of eccentric equatorial and circular inclined orbits, far more accurate than corresponding weak-field formulas for $(\dot{p}, \dot{e}, i)$. We use this technique to study the inspiral of a test body in inclined, eccentric Kerr orbits. Our results should be useful tools for constructing approximate waveforms that can be used to study data analysis problems for the future Laser Interferometer Space Antenna gravitational-wave observatory, in lieu of waveforms from more rigorous techniques that are currently under development.
\end{abstract}

DOI: 10.1103/PhysRevD.66.064005

PACS number(s): 04.30.Db, 04.25.Nx, 95.30.Sf

\section{BACKGROUND AND MOTIVATION}

The capture of stellar-mass compact objects by massive black holes residing in galactic nuclei is expected to be one of the most important sources of gravitational radiation for the future Laser Interferometer Space Antenna (LISA) spacebased detector $[1,2]$. Observing such events will provide information about stellar dynamics in galactic nuclei, and should make possible precise measurements of black hole masses and spins. Indeed, the waves generated by such a capture will encode a detailed description of the black hole's spacetime, making it possible to test whether the "large object" in the galactic nucleus is indeed a Kerr black hole as predicted by general relativity, or is some exotic massive compact object $[3,4]$.

Extracting such information will require accurate modeling of the gravitational waveform. The smallness of the system's mass ratio (typically, $\mu / M \sim 10^{-4}-10^{-6}$, where $\mu$ and $M$ are the masses for the captured body and the central hole, respectively) allows one to treat the small body as a "test particle" moving in the gravitational field of the black hole. In the absence of radiation, the small body moves on a geodesic orbit of the black hole [5]. These orbits have three integrals of motion (apart from $\mu$ ): energy $E$; angular momentum projected on the hole's spin axis, $L_{z}$; and Carter's third constant $Q$, related to the square of the angular momentum projected onto the equatorial plane. A body in a generic (eccentric and inclined) Kerr orbit traces an open ellipse precessing about the black hole's spin axis, resulting in a complicated overall motion. Astrophysical captured bodies will move in such complicated orbits.
The integrals of the motion are not constant in the presence of gravitational radiation-they evolve as energy and angular momentum are carried away by the waves. Because of the small mass ratio, they should change adiabatically, on time scales much longer than any orbital time scale. Hence, the orbit looks geodesic on short time scales. This fact can be used to calculate gravitational perturbations induced by the particle at infinity and at the hole's event horizon, using the Teukolsky-Sasaki-Nakamura formalism [6]. In this way, one can explicitly find the gravitational waveform at infinity and compute the corresponding fluxes of $E$ and $L_{z}$ to infinity and into the hole. If one also knows the evolution of the Carter constant, then the adiabatic nature of the inspiral allows one to treat the small body's motion as an evolution through a sequence of orbits: the body's world line $\mathbf{z}(t)$ is that of a geodesic orbit $\mathbf{z}_{\text {geod }}(t)$ whose orbital constants are slowly changing:

$$
\mathbf{z}(t)=\mathbf{z}_{\text {geod }}\left[t ; E(t), L_{z}(t), Q(t)\right] .
$$

Computing the inspiral properties is reduced to computing the parameter space trajectory $\left[E(t), L_{z}(t), Q(t)\right]$.

One can in fact infer the change in $Q$ and thus fix the small body's inspiral in two special cases: orbits that are equatorial, and orbits that are circular but inclined. A considerable amount of effort has been devoted to studying these orbits and their evolution due to gravitational-wave emission [7-12]. In these special cases, the evolution of the Carter constant $Q$ is constrained: it remains constant at $Q=0$ (equatorial orbits) or evolves such that the system's eccentricity is constant at $e=0$ (circular, inclined orbits) [13-15]. Accurate numerical computations, based on extracting $\dot{E}$ and $\dot{L}_{z}$ from 
fluxes of gravitational waves to infinity and down the event horizon, have detailed the effects of radiation reaction and the nature of gravitational-wave emission in these cases.

Unfortunately, this "flux-balancing" prescription fails in general-there is no known method for computing the rate $\dot{Q}$ from the gravitational-wave fluxes in the absence of special constraints. At the moment, the only applicable result is a weak-field, quadrupole-order calculation by Ryan [13], who used a weak-field radiation reaction force to infer $\dot{Q}$. Not surprisingly, Ryan's results become increasingly inaccurate and unreliable as the orbit comes closer to the black hole. It is likely that a strong-field gravitational self-force prescription will be needed to compute $\dot{Q}$. Many groups are now working on this problem [16]. It is generally acknowledged that no result applicable to strong-field Kerr orbits should be expected within the next few years. In the meantime, therefore, an investigation of possible approximation schemes for describing radiation reaction and wave emission by these orbits is highly desirable. Such schemes will play an important role in mapping out the scope of the data analysis task that the LISA community faces, making possible a realistic assessment of issues such as the amount of computing power needed, the accuracy with which black hole characteristics can be measured, and the difficulty of measuring signals if the inspiral rate is large enough to create a confusion-limited background [17].

In the remainder of this paper we present such an approximate scheme. The essential idea is to use the exact Kerr black hole geodesics to describe the system's dynamics, but to evolve through a sequence of those geodesics using the weak-field quadrupole-order fluxes for $\dot{E}$ and $\dot{L}_{z}$. Because this scheme mixes an exact notion of short-time-scale motion with an approximate description of the long-time-scale radiation effects, we call it a "hybrid" approximation. We find that the hybrid approximation faithfully reproduces features seen in the numerical strong-field analyses of radiation reaction. For example, we find that the rate of change of eccentricity will typically switch sign prior to plunging into the black hole; as a consequence, the orbit has substantial eccentricity near the end of inspiral. Self-consistent leading order calculations (which approximate the orbital dynamics as well as the radiation reaction) strongly underestimate this residual eccentricity. In some cases, they predict that the orbit is circular at the end of inspiral. This incorrect circularization could have a big effect on the waveform models that are used to lay the foundations of LISA data analysis, since a circular inspiral produces waves with less interesting harmonic structure than eccentric inspirals. We advocate this hybrid scheme as a method that is simple enough to produce waveforms that are "fast and dirty," but accurate enough to qualitatively reproduce features that should exist in real inspirals. We emphasize that the hybrid waveforms are not the ultimate models one would want to use as templates for analyzing the LISA data stream. Instead, we advocate them as tools for exploring issues in LISA data analysis, as described in the paragraph above.

The ideas behind the hybrid approximation and key equations are given in Sec. II; some of the more cumbersome details are presented in Appendix B. In Sec. III we then compare this technique's predictions to those of detailed numerical calculations for the two special cases that are wellunderstood now, equatorial orbits and inclined, circular orbits. We compare with the leading order results developed by Ryan [13], and show that the hybrid scheme qualitatively recovers features seen in the strong-field numerical calculations. Our results show that holding the inclination angle $\iota$ constant is more accurate than letting it evolve in the way that the weak-field fluxes "want" it to evolve (as compared to strong-field numerical calculations). We argue in Sec. III and Appendix A that this tells us that the "gravitational potential" felt by the inspiraling body is nearly spherical, and argue further that holding $\iota$ constant should work well for arbitrary orbits.

In Sec. IV we move to "generic" configurations, studying inspirals through a sequence of inclined, eccentric orbits. In most cases we find that the inspiral trajectories are qualitatively similar to inspiral in the equatorial plane. In particular, we find that most configurations plunge into the black hole with substantial residual eccentricity. We also map out the range of parameter space where we do not trust the hybrid approximation: when orbits reach too deeply into the strong field, or spiral in near inclination $90^{\circ}$, the weak-field fluxes that we use do not appear to be reliable. We conjecture in Sec. V on how an approximation could be developed to better understand the Carter constant's evolution. This approximation is based on the "zoom-whirl" behavior of strongfield eccentric orbits, recently described in Ref. [12]. We provide a concluding discussion and suggest directions for future work on this problem in Sec. VI. Throughout this paper, we use units in which $G=c=1$.

\section{THE HYBRID APPROXIMATION}

Generic Kerr geodesics can be parametrized by a triplet of constant orbital elements: the semi-latus rectum $p$, the eccentricity $e$, and the inclination angle $\iota$. The elements $p$ and $e$ define the orbit's radial turning points, the apastron and periastron:

$$
r_{a}=\frac{p}{1-e}, \quad r_{p}=\frac{p}{1+e} .
$$

In the strong field of a Kerr black hole, there are many ways that one could define an "inclination angle" - for example, the turning points of the orbit's latitudinal motion, or the angle at which the small body crosses the equator as seen by distant observers. We use the following definition:

$$
\cos \iota=\frac{L_{z}}{\sqrt{Q+L_{z}^{2}}} .
$$

This definition does not correspond to either of these examples, but is very convenient: it depends simply on orbital constants and has a useful intuitive description, suggesting that the Carter constant $Q$ is essentially just the square of the angular momentum projected into the equatorial plane. (This description is in fact exactly correct for Schwarzschild black 
holes; for non-zero spin it is not quite correct, but is good enough to be useful. We discuss this issue in more detail in Appendix A.) The orbital elements can be written as functions of $\left(E, L_{z}, Q\right)$, and vice versa. Consequently, we can write their time derivatives as $\dot{p}=\dot{p}\left(p, e, \iota, \dot{E}, \dot{L}_{z}, \dot{Q}\right)$, and similarly for $\dot{e}$ and $i$.

As already mentioned, we do not yet know how to accurately calculate $\dot{Q}$. It is only known to leading order in $M / p$ and in the spin of the black hole [13]. The orbital parameters used in Ref. [13] are an eccentricity $\bar{e}$ (different from $e$ ), a semi-major axis $\bar{a}$, and an inclination angle $\iota$ (identical to our $\iota$ ). The two sets of parameters are related by

$$
\begin{aligned}
1-e^{2} & =\left(1-\bar{e}^{2}\right)\left[1-\frac{4 a}{M}\left(\frac{M}{p}\right)^{3 / 2} e^{2} \cos \iota\right], \\
p & =\bar{a}\left(1-e^{2}\right)\left[1-\frac{2 a}{M}\left(\frac{M}{p}\right)^{3 / 2} e^{2} \cos \iota\right] .
\end{aligned}
$$

The parametrizations are consistent in the weak field, and are identical for zero spin. Rewriting Ryan's fluxes in terms of our parameters yields

$$
\begin{aligned}
\dot{E}= & -\frac{32}{5} \frac{\mu^{2}}{M^{2}}\left(\frac{M}{p}\right)^{5}\left(1-e^{2}\right)^{3 / 2} \\
& \times\left[f_{1}(e)-\frac{a}{M}\left(\frac{M}{p}\right)^{3 / 2} \cos \iota f_{2}(e)\right], \\
\dot{L}_{z}= & -\frac{32}{5} \frac{\mu^{2}}{M}\left(\frac{M}{p}\right)^{7 / 2}\left(1-e^{2}\right)^{3 / 2}\left[\cos \iota f_{3}(e)\right. \\
& \left.+\frac{a}{M}\left(\frac{M}{p}\right)^{3 / 2}\left[f_{4}(e)-\cos ^{2} \iota f_{5}(e)\right]\right], \\
\dot{C}= & -\frac{64}{5} \mu^{3}\left(\frac{M}{p}\right)^{3}\left(1-e^{2}\right)^{3 / 2} \\
& \times\left[f_{3}(e)-\frac{a}{M}\left(\frac{M}{p}\right)^{3 / 2} \cos \iota f_{6}(e)\right],
\end{aligned}
$$

where $C \equiv Q+L_{z}^{2}$, and

$$
\begin{aligned}
& f_{1}(e)=1+\frac{73}{24} e^{2}+\frac{37}{96} e^{4}, \\
& f_{2}(e)=\frac{73}{12}+\frac{823}{24} e^{2}+\frac{949}{32} e^{4}+\frac{491}{192} e^{6}, \\
& f_{3}(e)=1+\frac{7}{8} e^{2}, \\
& f_{4}(e)=\frac{61}{24}+\frac{63}{8} e^{2}+\frac{95}{64} e^{4}, \\
& f_{5}(e)=\frac{61}{8}+\frac{91}{4} e^{2}+\frac{461}{64} e^{4},
\end{aligned}
$$

$$
f_{6}(e)=\frac{97}{12}+\frac{37}{2} e^{2}+\frac{211}{32} e^{4}
$$

In the $a=0$ limit, Eqs. (2.5) and (2.6) reduce to the celebrated Peters-Mathews formulas [18].

The rates $\dot{q}_{j}=\{\dot{p}, \dot{e}, \dot{i}\}$ can be written

$$
\dot{q}_{j}=H^{-1}\left(b_{j} \dot{E}+c_{j} \dot{L}_{z}+d_{j} \dot{Q}\right) .
$$

The quantities $H$ and $b_{j}, c_{j}, d_{j}$ are all constructed in a straightforward way from derivatives of $E, L_{z}, Q$ with respect to $p, e, \iota$; the resulting expressions are rather cumbersome, and so are written out in Appendix B. We emphasize that these functions encode the exact geodesic motion.

The main idea behind the hybrid scheme is simple: calculate the time derivatives $\dot{q}_{j}$ using the exact coefficients $b_{j}$, $c_{j}, d_{j}$ and the approximate fluxes (2.5)-(2.7). A consistent leading-order calculation (that is, leading order in $M / p$ and $a / M$ ) would instead approximate the coefficients along with the fluxes. Knowing the rates $\dot{q}_{j}$ makes it possible to build the parameter space trajectories $q_{j}(t)$ followed by a small body spiraling into a black hole: given initial values $q_{j}(0)$, one simply "integrates up" the derivatives $\dot{q}_{j}$ to generate the inspiral trajectory. For example, a simple-minded Eulermethod integration would step from parameter space coordinates $\left(t, q_{j}\right)$ to $\left(t+\delta t, q_{j}+\dot{q}_{j} \delta t\right)$. Generalization to more sophisticated integration techniques is straightforward. The trajectories $q_{j}(t)$ are the main result of this paper. From them, it is a simple matter to compute quantities such as the gravitational waveform generated during an inspiral, and thus to begin testing ideas more directly related to data analysis. We will not develop such waveforms here, but will instead defer them to a later analysis.

\section{COMPARISON WITH NUMERICAL RESULTS}

The reliability of this method can be assessed by applying it to specific families of orbits where accurate numerical results are already known. We first consider equatorial eccentric orbits, recently studied by Glampedakis and Kennefick [12]. Such orbits always have $\iota=0^{\circ}$ (prograde) or $\iota=180^{\circ}$ (retrograde), leaving $p$ and $e$ as unspecified parameters. Equation (2.14) becomes

$$
\begin{aligned}
& \dot{p}=H_{\mathrm{eq}}^{-1}\left(-E_{, e} \dot{L}_{z}+L_{z, e} \dot{E}\right), \\
& \dot{e}=H_{\mathrm{eq}}^{-1}\left(E_{, p} \dot{L}_{z}-L_{z, p} \dot{E}\right),
\end{aligned}
$$

where $H_{\mathrm{eq}}=E_{, p} L_{z, e}-E_{, e} L_{z, p}$. The leading-order approximation for these expressions is [8]

$$
\begin{aligned}
& \dot{p}=-\frac{64}{5} \frac{\mu}{M}\left(1-e^{2}\right)^{3 / 2}\left(\frac{M}{p}\right)^{3}\left(1+\frac{7}{8} e^{2}\right), \\
& \dot{e}=-\frac{304}{15} \frac{\mu}{M^{2}} e\left(1-e^{2}\right)^{3 / 2}\left(\frac{M}{p}\right)^{4}\left(1+\frac{121}{304} e^{2}\right) .
\end{aligned}
$$



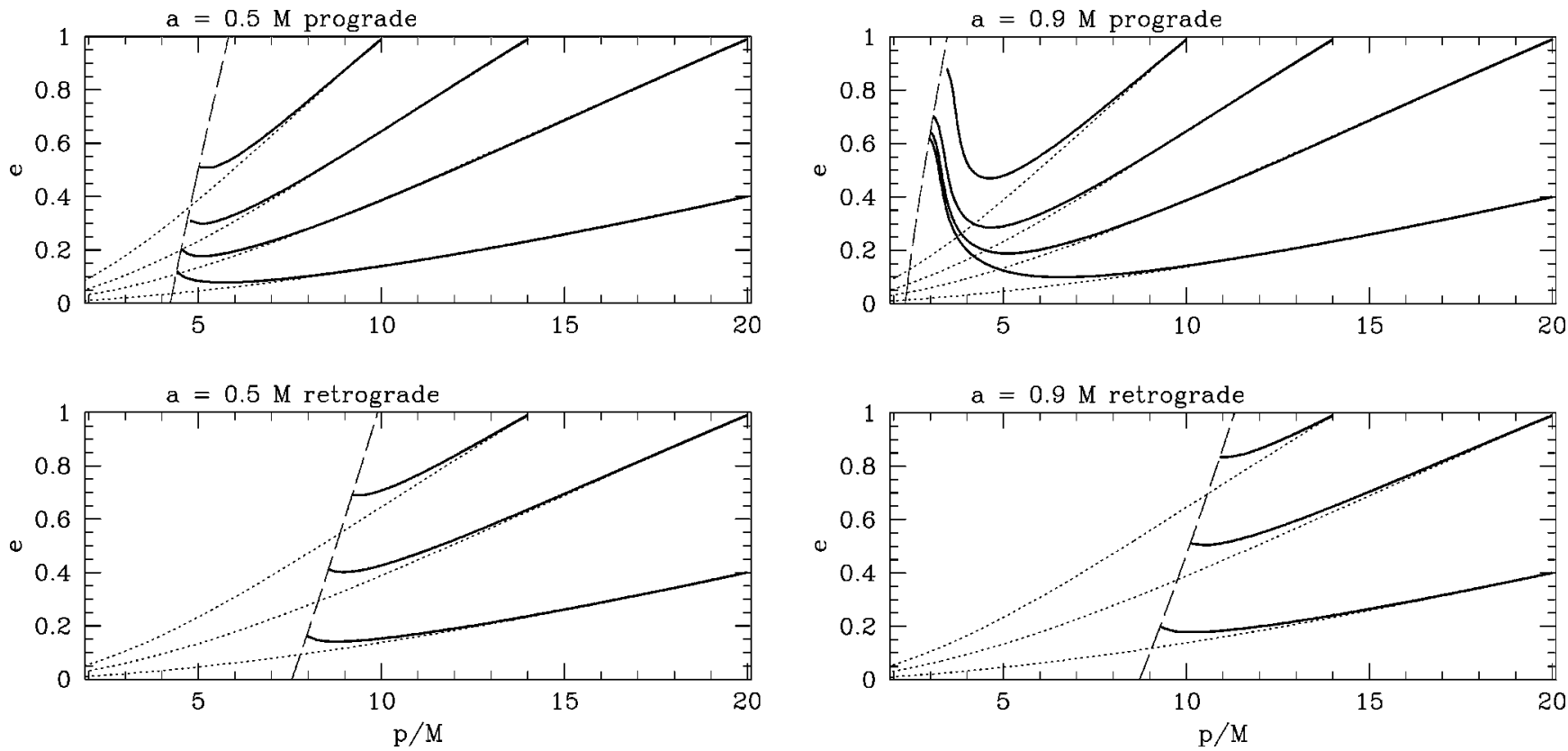

FIG. 1. Comparing equatorial inspiral. We show inspiral into a hole with spin $a=0.5 M$ (left panel) and into a hole with $a=0.9 M$ (right panel). In each panel, the top half shows prograde inspirals and the bottom retrograde inspirals. In each set, the dashed line represents the separatrix separating stable from unstable orbits. We used the hybrid approximation discussed in the text to radiatively evolve orbits with initial parameters $\left(p_{i}, e_{i}\right)=(20 M, 0.4),(20 M, 0.99),(14 M, 0.99)$, and $(10 M, 0.99)$. The inspiral trajectories are shown as the heavy lines in each plot. (The final set is not included in the retrograde inspirals since the initial conditions are not stable in those cases.) The dotted trajectories in each plot show the leading-order predictions generated using Eq. (3.5). Note the significant qualitative difference between the two calculations at the vicinity of each separatrix. Note also the extremely large growth in eccentricity seen in the prograde inspirals for $a=0.9 M$ just before reaching the separatrix. Comparison with accurate strong-field numerical results shows that this growth is excessive.

Note that we could equally well use the corresponding expressions with the leading-order spin terms included (see [12]), but it turns out that they essentially give the same results as Eqs. (3.3) and (3.4). These equations can be combined to give a simple expression that describes the orbital evolution on the $p$-e plane:

$$
p(e)=p_{i}\left(\frac{e}{e_{i}}\right)^{12 / 19}\left[\frac{1+121 e^{2} / 304}{1+121 e_{i}^{2} / 304}\right]^{870 / 2299},
$$

where $p_{i}$ and $e_{i}$ are initial values. We are now ready to compare the inspiral trajectories generated by Eq. (3.5) with those obtained by the hybrid scheme.

Representative results for astrophysically relevant initial parameters are shown in Fig. 1. We compare the leadingorder trajectories found using Eq. (3.5) (dotted lines) with the trajectories predicted by the hybrid scheme (solid lines). Note that the time dependence of the inspiral is suppressed in this figure: most time is actually spent at large $p$. The total duration of an inspiral scales with $M^{2} / \mu$. The shape of a curve, however, does not depend on this ratio, provided that the mass ratio is extreme: these curves are universal for $\mu$ $\ll M$. We show inspiral for both prograde and retrograde orbits, for black hole spins $a=0.5 M$ and $a=0.9 M$.

In all cases, the hybrid and the leading-order calculations agree for $p \gg M$, as expected. Differences between the two methods become apparent in the strong field. The leadingorder inspiral trajectory exhibits constantly decreasing eccen- tricity. This is in marked contrast with the rigorous strongfield calculations (numerical and analytical) of Refs. [7,8,12] showing that there exists a region near the separatrix between stable and unstable orbits where $\dot{e}$ reverses sign: the eccentricity should grow near the separatrix. It is very encouraging that the eccentricity does in fact grow when the hybrid approximation is used. Moreover, the location of the critical points in these curves where $\dot{e}=0$ is in good agreement (at the order of a few percent) with the numerical results of Refs. [8,12]; see Table I. Three of the four cases shown in Fig. 1 appear "good" in the sense that the trajectories appear to agree reasonably well with what we expect based on strong-field numerical analyses (cf. Ref. [12]). The same comparison for the fourth case $(a=0.9 M$, prograde; upper plot in the right-hand panel of Fig. 1) reveals that both the eccentricity growth near the separatrix and the distance of the critical curve $\dot{e}=0$ from the separatrix are excessive. Prograde orbits of rapidly rotating black holes reach rather deep into the black hole's strong field where the weak-field fluxes (2.5) and (2.6) cannot be trusted. As we shall see when we move on to generic inspirals (Sec. IV), this breakdown of the weak-field flux formulas means that the hybrid approximation does not accurately describe the inspiral of shallow inclination orbits $\left(\iota \leqslant 20^{\circ}\right.$ or so) into rapidly rotating black holes ( $a \gtrsim 0.85 M$ or so).

It is possible to get some insight into the superior qualitative description of the inspiral in the strong field region given by our approach. The phenomenon of orbital circular- 
TABLE I. Comparing critical curve values $p_{\text {crit }}$ for equatorial eccentric orbits. These are the $p$ values at which the eccentricity evolution switches sign, beginning to grow rather than shrink. We show the values of $p_{\text {crit }}$ calculated numerically $[8,12]$ (third column) and using the hybrid approximation (fourth column), for a variety of black hole spins (negative $a / M$ represents retrograde orbits) and eccentricities. The fifth column shows the fractional difference between the numerical and the approximate results (approximate - numerical)/(numerical).

\begin{tabular}{lcccc}
\hline \hline$a / M$ & $e$ & $\begin{array}{c}p_{\text {crit }} / M \\
\text { (numerical) }\end{array}$ & $\begin{array}{c}p_{\text {crit }} / M \\
\text { (hybrid approx.) }\end{array}$ & $\begin{array}{c}\text { Fractional } \\
\text { difference }\end{array}$ \\
\hline 0 & 0.2 & 6.76 & 6.92 & 0.0237 \\
0 & 0.4 & 6.99 & 7.13 & 0.0200 \\
0.5 & 0.3 & 4.85 & 5.06 & 0.0433 \\
0.5 & 0.5 & 5.08 & 5.21 & 0.0250 \\
-0.99 & 0.3 & 10.25 & 10.53 & 0.0273 \\
-0.99 & 0.5 & 10.59 & 10.78 & 0.0179 \\
\hline \hline
\end{tabular}

ization as a result of some form of dissipation is seen in many astrophysical situations, such as that of satellites whose orbits are decaying due to atmospheric friction. The reason is that the dissipating mechanism causes the particle to "drop" in its potential well, the usual geometry of which ensures that the orbital eccentricity decreases. In our case another mechanism becomes significant as the unstable plunge orbit is approached at the end of the inspiral. As this occurs the potential becomes shallower (as the minimum turns into a saddle point at plunge), and this tends to increase the eccentricity of the orbit. Shortly before plunge this mechanism overcomes the circularizing tendency. It is not surprising that the hybrid approximation can qualitatively replicate the eccentricity increasing behavior, because it exactly describes the shape of the orbital potential, which is so critical to this effect.

Table II compares data for $\dot{p}$ and $\dot{e}$. In this sample, the hybrid approach clearly is more accurate than the leadingorder approximation. This comparison is a very strict test of the accuracy of this scheme. As discussed above, we believe that the hybrid approximation is reliable as long as $r_{p}$ $\gtrsim 5 \mathrm{M}$. The weak-field fluxes that we use cannot be trusted very deep in the strong field - the spin correction terms in Eqs. (2.5) and (2.6) dominate the leading order term. The method therefore fails when we push to smaller $r_{p}$. This effectively constrains the black hole spin to $a \leqq 0.5 M$ for prograde motion - for larger spins, the innermost stable orbit and hence $r_{p}$ come too close to the horizon. For retrograde orbits, the results are much more accurate since $r_{p}$ never comes close to the horizon, regardless of the spin. Finally, we emphasize the essential role Ryan's fluxes (2.5) and (2.6) play in calculating $\dot{p}$ and $\dot{e}$. Had we used instead the Peters-Mathews fluxes, the resulting inspirals would predict a rapid circularization under radiation reaction: we find that the Peters-Mathews fluxes reduce the eccentricity to zero well before reaching the saddle point of the orbital potential, and so the eccentricity never grows. This is in sharp disagreement with the numerical results.

A major prediction of the hybrid approximation is that for equatorial orbits the residual eccentricity prior to plunge should be substantial, in strong contrast to the prediction of the leading order formula (3.5). In many cases, the leading order results predict that the orbit will actually circularize prior to plunge. Because the harmonic structure of a circular inspiral is rather different from that of an inspiral with substantial eccentricity, these results have strong implications for the waveform models to be used in LISA's data analysis.

We next consider circular inclined orbits, which were recently studied by Hughes [10]. One of the most important findings of Ref. [10] is that the angle $\iota$ remains almost constant during inspiral, even when the particle is crossing strong field regions.

For these orbits, the rates $\dot{p}$ and $i$ are given by

$$
\begin{gathered}
\dot{p}=H_{\text {circ }}^{-1}\left(-L_{z, \iota} \dot{E}+E_{, \iota} \dot{L}_{z}\right), \\
i=H_{\text {circ }}^{-1}\left(L_{z, p} \dot{E}-E_{, p} \dot{L}_{z}\right),
\end{gathered}
$$

where $H_{\text {circ }}=E_{, \iota} L_{z, p}-L_{z, \iota} E_{, p}$. In order to obtain these formulas we first expressed $\dot{Q}$ in terms of $\dot{E}$ and $\dot{L}_{z}$ making use of the "circular goes to circular" theorems [13-15]; see Ref. [11] for further discussion. The leading-order expression for $i$ is [19]

$$
i=\frac{244}{15} \frac{\mu}{M^{2}} \frac{a}{M}\left(\frac{M}{p}\right)^{11 / 2} \sin \iota
$$

$\dot{p}$ follows from Eq. (3.3), setting $e=0$.

Table III compares data for $\dot{p}$ and $i$ using the hybrid approximation to the the results obtained using Eqs. (3.3) and (3.8), together with accurate numerical results from Ref. [10]. Figure 2 shows inspirals of circular inclined orbits with our method and using the leading-order formulas. Both approximations predict that $\iota$ changes in such a way as to drive the orbit to an equatorial retrograde configuration (that is, $\iota$ increases). The two calculations agree at large radii. In the strong field, the leading-order formulas break down - the inclination angle tends to increase dramatically. The behavior of the hybrid-scheme inspiral is more reasonable.

Although the hybrid scheme is much better behaved in the strong field, the growth of $\iota$ we see is still quite a bit larger than detailed numerical calculations predict $[10,11]$. Based on those numerical results, a more accurate scheme would be to simply require that $\iota$ remain constant. Combining $d \iota / d t$ $=0$ with Eq. (2.2) yields the rule

$$
\dot{Q}=\frac{2 Q}{L_{z}} \dot{L}_{z}
$$

This rule is consistent with our description of $Q$ as roughly the squared component of angular momentum projected into the equatorial plane. If the spacetime is perfectly spherical (i.e., Schwarzschild black holes), then $Q$ is exactly such an angular momentum component: $Q_{\text {spherical }} \equiv L_{x}^{2}+L_{y}^{2}$. It is easy to show that an inspiral in this spacetime would proceed at exactly constant inclination angle: gravitational waves carry off exactly the right amounts of $L_{x}$ and $L_{y}$ to hold $\iota$ constant, 
TABLE II. Comparing the rates $\dot{p}, \dot{e}$ for several equatorial eccentric orbits. The fifth column in this table shows $\dot{p} ;$ the seventh column shows $\dot{e}$. Within each section of the table, the first row of columns five and seven contains accurate numerical data from [8,12], the second row shows data using the hybrid scheme outlined in this paper, and the third row shows data using quadrupole order results. The sixth and eighth columns show the fractional differences between the two approximation schemes and the accurate numerical results. In all cases but one, the hybrid approximation is closer to the accurate numerical calculation, sometimes substantially so.

\begin{tabular}{|c|c|c|c|c|c|c|c|}
\hline$a / M$ & $p / M$ & $e$ & Calculation & $(M / \mu) \dot{p}$ & Frac. diff. in $\dot{p}$ & $\left(M^{2} / \mu\right) \dot{e}$ & Frac. diff. in $\dot{e}$ \\
\hline \multirow[t]{3}{*}{0} & 7.505 & 0.189 & Numerical & $-7.475 \times 10^{-2}$ & - & $-1.967 \times 10^{-3}$ & - \\
\hline & & & Hybrid & $-6.859 \times 10^{-2}$ & 0.0824 & $-1.291 \times 10^{-3}$ & 0.3434 \\
\hline & & & Leading order & $-2.957 \times 10^{-2}$ & 0.6044 & $-1.159 \times 10^{-3}$ & 0.4108 \\
\hline \multirow[t]{3}{*}{0} & 6.9 & 0.4 & Numerical & $-4.240 \times 10^{-1}$ & - & $+1.047 \times 10^{-2}$ & - \\
\hline & & & Hybrid & $-3.056 \times 10^{-1}$ & 0.2792 & $+1.506 \times 10^{-2}$ & -0.4384 \\
\hline & & & Leading order & $-3.420 \times 10^{-2}$ & 0.9193 & $-2.929 \times 10^{-3}$ & 1.2797 \\
\hline \multirow[t]{3}{*}{0.5} & 6.5 & 0.4 & Numerical & $-5.999 \times 10^{-2}$ & - & $-5.155 \times 10^{-3}$ & - \\
\hline & & & Hybrid & $-4.606 \times 10^{-2}$ & 0.2322 & $-3.356 \times 10^{-3}$ & 0.3490 \\
\hline & & & Leading order & $-4.091 \times 10^{-2}$ & 0.3181 & $-3.719 \times 10^{-3}$ & 0.2786 \\
\hline \multirow[t]{3}{*}{0.5} & 15 & 0.4 & Numerical & $-3.371 \times 10^{-3}$ & - & $-1.341 \times 10^{-4}$ & - \\
\hline & & & Hybrid & $-3.358 \times 10^{-3}$ & 0.0039 & $-1.334 \times 10^{-4}$ & 0.0052 \\
\hline & & & Leading order & $-3.328 \times 10^{-3}$ & 0.0128 & $-1.311 \times 10^{-4}$ & 0.0224 \\
\hline \multirow[t]{3}{*}{0.5} & 4.8 & 0.3 & Numerical & $-6.354 \times 10^{-1}$ & - & $+1.369 \times 10^{-2}$ & - \\
\hline & & & Hybrid & $-4.858 \times 10^{-1}$ & 0.2354 & $+3.519 \times 10^{-2}$ & -1.5705 \\
\hline & & & Leading order & $-4.849 \times 10^{-2}$ & 0.9237 & $-4.432 \times 10^{-3}$ & 1.3237 \\
\hline \multirow[t]{3}{*}{0.9} & 5 & 0.4 & Numerical & $-7.507 \times 10^{-2}$ & - & $-9.266 \times 10^{-3}$ & - \\
\hline & & & Hybrid & $-4.617 \times 10^{-2}$ & 0.3850 & $-1.965 \times 10^{-3}$ & 0.7879 \\
\hline & & & Leading order & $-2.698 \times 10^{-3}$ & 0.9641 & $-9.732 \times 10^{-5}$ & 0.9895 \\
\hline \multirow[t]{3}{*}{-0.5} & 10 & 0.4 & Numerical & $-3.115 \times 10^{-2}$ & - & $-1.379 \times 10^{-3}$ & - \\
\hline & & & Hybrid & $-2.494 \times 10^{-2}$ & 0.1993 & $-9.107 \times 10^{-4}$ & 0.3396 \\
\hline & & & Leading order & $-1.337 \times 10^{-2}$ & 0.5708 & $-7.931 \times 10^{-4}$ & 0.4249 \\
\hline \multirow[t]{3}{*}{-0.99} & 10.5 & 0.4 & Numerical & $-7.516 \times 10^{-2}$ & - & $-5.312 \times 10^{-4}$ & - \\
\hline & & & Hybrid & $-5.506 \times 10^{-2}$ & 0.2674 & $-5.223 \times 10^{-4}$ & 0.0168 \\
\hline & & & Leading order & $-9.704 \times 10^{-3}$ & 0.8709 & $-5.461 \times 10^{-4}$ & 0.0281 \\
\hline \multirow[t]{3}{*}{-0.99} & 15 & 0.4 & Numerical & $-5.766 \times 10^{-3}$ & - & $-2.141 \times 10^{-4}$ & - \\
\hline & & & Hybrid & $-5.295 \times 10^{-3}$ & 0.0817 & $-1.875 \times 10^{-4}$ & 0.1242 \\
\hline & & & Leading order & $-3.328 \times 10^{-3}$ & 0.4228 & $-1.311 \times 10^{-4}$ & 0.3877 \\
\hline
\end{tabular}

so Eq. (3.9) is exactly correct in this case. One would expect $\iota$ to remain nearly constant if the spacetime does not deviate too strongly from sphericity. Rigorous numerical results for the circular inclined case show that $\iota$ indeed remains nearly constant; it thus appears that the Kerr metric is not too aspherical over much of the inspiral (modulo frame dragging). Additional evidence for the validity of this statement is given by the discussion in Appendix A. Since the orbit's eccentricity does not enter this argument at all, it is likely that Eq. (3.9) will work well for inclined eccentric orbits also.

\section{EVOLVING GENERIC ORBITS}

Having established the reliability and limitations of the hybrid scheme, we move to the main subject of this paper: the study of inspirals of test bodies in generic orbits where only leading-order results are currently available [13]. We began this analysis employing Ryan's fluxes, Eqs. (2.5)(2.7), but quickly faced disappointing results. We found that hybrid-scheme inspirals produced with these fluxes did not behave well far from the two limits discussed above, particularly in the strong field. For example, the eccentricity tended 
TABLE III. Comparing the rates $\dot{p}, i$ for several inclined circular orbits. The fifth column shows $\dot{p}$; the seventh column shows $i$. Within each section of the table, the first row of columns five and seven contains accurate numerical data from [10], the second row shows data using the hybrid scheme outlined in this paper, and the third row shows data using leading order results. The sixth and eighth columns show the fractional differences between the two approximation schemes and the accurate numerical results. In most cases in this sample, the hybrid scheme performs much better than the leading-order approximation when compared to the rigorous numerical data. The only case which this is not true is for $i$ of retrograde orbits. Nevertheless, this small inaccuracy has no impact on the calculation of generic inspirals, as we assume that $\iota=$ const. Note the enormous difference between the numerical and the leading order results in the table's final entry. This is because that point is fairly close to the separatrix between stable and unstable orbits. Since the leading-order calculation has no notion of this separatrix, it is particularly inaccurate here.

\begin{tabular}{|c|c|c|c|c|c|c|c|}
\hline$a / M$ & $p / M$ & $\iota$ (degrees) & Calculation & $(M / \mu) \dot{p}$ & Frac. diff. in $\dot{p}$ & $\left(M^{2} / \mu\right) i$ & Frac. diff. in $i$ \\
\hline 0.95 & 7 & 62.43 & $\begin{array}{c}\text { Numerical } \\
\text { Hybrid } \\
\text { Leading order }\end{array}$ & $\begin{array}{l}-4.657 \times 10^{-2} \\
-4.497 \times 10^{-2} \\
-2.750 \times 10^{-2}\end{array}$ & $\begin{array}{c}- \\
0.0344 \\
0.4095\end{array}$ & $\begin{array}{l}1.207 \times 10^{-4} \\
2.639 \times 10^{-4} \\
3.080 \times 10^{-4}\end{array}$ & $\begin{array}{c}- \\
1.1864 \\
1.5518\end{array}$ \\
\hline 0.05 & 7 & 60.17 & $\begin{array}{c}\text { Numerical } \\
\text { Hybrid } \\
\text { Leading order }\end{array}$ & $\begin{array}{l}-1.096 \times 10^{-1} \\
-1.048 \times 10^{-1} \\
-3.676 \times 10^{-2}\end{array}$ & $\begin{array}{c}- \\
0.0438 \\
0.6642\end{array}$ & $\begin{array}{l}1.087 \times 10^{-5} \\
1.207 \times 10^{-5} \\
1.587 \times 10^{-5}\end{array}$ & $\begin{array}{c}- \\
0.1104 \\
0.4500\end{array}$ \\
\hline 0.5 & 10 & 67.56 & $\begin{array}{c}\text { Numerical } \\
\text { Hybrid } \\
\text { Leading order }\end{array}$ & $\begin{array}{l}-1.583 \times 10^{-2} \\
-1.645 \times 10^{-2} \\
-1.194 \times 10^{-2}\end{array}$ & $\begin{array}{c}- \\
0.0392 \\
0.2457\end{array}$ & $\begin{array}{l}1.546 \times 10^{-5} \\
2.043 \times 10^{-5} \\
2.377 \times 10^{-5}\end{array}$ & $\begin{array}{c}- \\
0.3215 \\
0.5375\end{array}$ \\
\hline 0.5 & 10 & 126.76 & $\begin{array}{c}\text { Numerical } \\
\text { Hybrid } \\
\text { Leading order }\end{array}$ & $\begin{array}{l}-2.329 \times 10^{-2} \\
-2.341 \times 10^{-2} \\
-1.414 \times 10^{-2}\end{array}$ & $\begin{array}{c}- \\
0.0051 \\
0.3929\end{array}$ & $\begin{array}{l}1.892 \times 10^{-5} \\
1.643 \times 10^{-5} \\
2.060 \times 10^{-5}\end{array}$ & $\begin{array}{c}- \\
0.1316 \\
0.0888\end{array}$ \\
\hline 0.9 & 10 & 74.07 & $\begin{array}{c}\text { Numerical } \\
\text { Hybrid } \\
\text { Leading order }\end{array}$ & $\begin{array}{l}-1.544 \times 10^{-2} \\
-1.567 \times 10^{-2} \\
-1.169 \times 10^{-2}\end{array}$ & $\begin{array}{c}-\overline{-} \\
0.0149 \\
0.2429\end{array}$ & $\begin{array}{l}2.715 \times 10^{-5} \\
3.857 \times 10^{-5} \\
4.452 \times 10^{-5}\end{array}$ & $\begin{array}{c}- \\
0.4206 \\
0.6398\end{array}$ \\
\hline 0.9 & 10 & 131.57 & $\begin{array}{c}\text { Numerical } \\
\text { Hybrid } \\
\text { Leading order }\end{array}$ & $\begin{array}{l}-3.253 \times 10^{-2} \\
-3.082 \times 10^{-2} \\
-1.545 \times 10^{-2}\end{array}$ & $\begin{array}{c}- \\
0.0526 \\
0.5250\end{array}$ & $\begin{array}{l}3.887 \times 10^{-5} \\
2.612 \times 10^{-5} \\
3.464 \times 10^{-5}\end{array}$ & $\begin{array}{c}- \\
0.3280 \\
0.1088\end{array}$ \\
\hline 0.5 & 6 & 48.33 & $\begin{array}{c}\text { Numerical } \\
\text { Hybrid } \\
\text { Leading order }\end{array}$ & $\begin{array}{l}-1.237 \times 10^{-1} \\
-1.135 \times 10^{-1} \\
-4.440 \times 10^{-2}\end{array}$ & $\begin{array}{c}- \\
0.0824 \\
0.6411\end{array}$ & $\begin{array}{l}1.410 \times 10^{-4} \\
2.614 \times 10^{-4} \\
3.190 \times 10^{-4}\end{array}$ & $\begin{array}{c}- \\
0.8539 \\
1.2624\end{array}$ \\
\hline 0.5 & 6 & 67.81 & $\begin{array}{c}\text { Numerical } \\
\text { Hybrid } \\
\text { Leading order }\end{array}$ & $\begin{array}{l}-2.020 \times 10^{-1} \\
-1.779 \times 10^{-1} \\
-5.082 \times 10^{-2}\end{array}$ & $\begin{array}{c}- \\
0.1193 \\
0.7484\end{array}$ & $\begin{array}{l}2.094 \times 10^{-4} \\
2.992 \times 10^{-4} \\
3.954 \times 10^{-4}\end{array}$ & $\begin{array}{c}- \\
0.4288 \\
0.8882\end{array}$ \\
\hline 0.9 & 6 & 54.64 & $\begin{array}{c}\text { Numerical } \\
\text { Hybrid } \\
\text { Leading order }\end{array}$ & $\begin{array}{l}-7.846 \times 10^{-2} \\
-6.950 \times 10^{-2} \\
-3.598 \times 10^{-2}\end{array}$ & $\begin{array}{c}- \\
0.1142 \\
0.5674\end{array}$ & $\begin{array}{l}2.015 \times 10^{-4} \\
5.486 \times 10^{-4} \\
6.268 \times 10^{-4}\end{array}$ & $\begin{array}{c}- \\
1.7226 \\
2.1107\end{array}$ \\
\hline 0.9 & 6 & 99.55 & $\begin{array}{c}\text { Numerical } \\
\text { Hybrid } \\
\text { Leading order }\end{array}$ & $\begin{array}{c}-74.32 \\
-48.02 \\
-6.593 \times 10^{-2}\end{array}$ & $\begin{array}{c}- \\
0.3539 \\
0.9991\end{array}$ & $\begin{array}{l}6.337 \times 10^{-4} \\
5.241 \times 10^{-4} \\
7.580 \times 10^{-4}\end{array}$ & $\begin{array}{c}- \\
0.1729 \\
0.1961\end{array}$ \\
\hline
\end{tabular}

to grow extremely large very rapidly in some cases. The root of the problem lies in the expression for the $\dot{Q}$ flux, Eq. (2.7), which apparently is not as accurate as we would require it to be. The qualitative behavior of our inspirals is more reasonable when the rule given by Eq. (3.9) is used to compute $\dot{Q}$ instead, forcing $\iota$ to be constant. Following the discussion at the end of Sec. III and in Appendix A, it is likely that this rule is accurate enough for our purposes anyway, and so we shall use it from this point onward. In all likelihood, detailed self-force calculations will be needed to test the accuracy of 

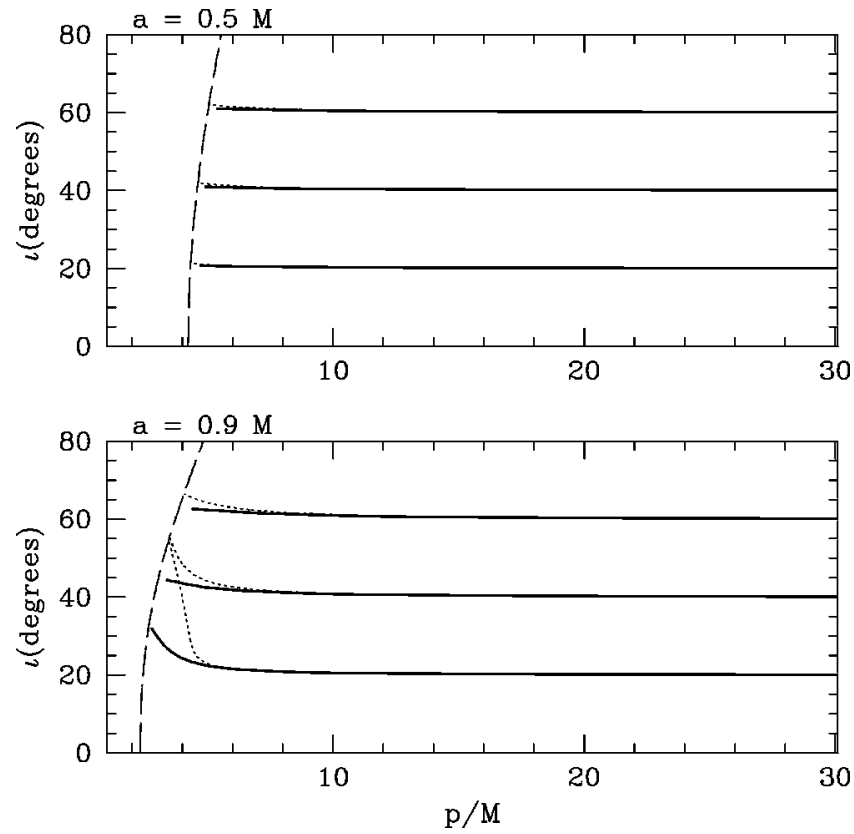

FIG. 2. Comparing circular, inclined inspiral. We show inspiral into a hole with spin $a=0.5 M$ (top) and spin $a=0.9 M$ (bottom). The solid lines show inspiral using the hybrid approximation; the dotted lines show the leading order inspiral prediction. The dashed curve shows the separatrix between stable and unstable orbits. Both approximations show that the inclination angle increases, especially right before reaching the separatrix. However, the increase predicted by the leading-order prediction is far too large, particularly for rapidly spinning black holes. The inspiral predicted by the hybrid approximation is closer to what is seen in rigorously computed inspirals. Nonetheless, it too shows an increase in $\iota$ that is probably excessive. As we argue in the text, holding $\iota$ constant produces an inspiral sequence that is probably closest of all to strong-field calculations and should be acceptably accurate.

the constant inclination rule.

Using the fluxes given in Eqs. (2.5) and (2.6) with the constant- $\iota$ rule Eq. (3.9) produces inspirals that agree with the leading-order results when $p \gg M$, which smoothly converge to the equatorial case for $\iota \rightarrow 0^{\circ}$ and $\iota \rightarrow 180^{\circ}$ and that exhibit an $\dot{e}>0$ region near the separatrix. Examples of this behavior are shown in Fig. 3 (spin $a=0.5 M$ ) and Fig. 4 (spin $a=0.9 M)$. Several interesting features can be seen in these figures. The trajectories for $\iota<90^{\circ}$ are qualitatively similar to the equatorial, prograde trajectories shown in Fig. 1. In particular, each such trajectory passes through a critical point at which $\dot{E}=0$ after which eccentricity grows. The system typically has substantial non-zero eccentricity when it reaches the separatrix. Also, note the excessive growth in eccentricity near the separatrix for $a=0.9 \mathrm{M}$ and $\iota=30^{\circ}$. At shallow inclination angle, the separatrix is very deep in the black hole's strong field, so the inspiral proceeds to small $r$ before plunging. Just as in the case of equatorial orbits for $a=0.9 M$, the weak-field flux formulas that we use cannot be trusted this far into the Kerr black hole strong field.

The qualitative appearance of the inspirals for $\iota>90^{\circ}$ is quite a bit different from the $\iota<90^{\circ}$ inspirals. Looking at the right hand sides of Figs. 3 and 4, we see that the hybrid approximation predicts that many of these inspirals completely circularize prior to reaching the separatrix. We do not believe that this behavior is robust. Indeed, we find that the behavior of inspirals exhibits a rather sharp transition as the inclination angle goes from slightly below $90^{\circ}$ to slightly above. This behavior arises from the cos $\iota$ terms in Eqs. (2.5) and (2.6), which switch sign at this transition. We thus do not believe that this rapid circularization is physical, but instead attribute it to poor behavior of the hybrid approximation at $\iota \gtrsim 90^{\circ}$.

Having established that the behavior of hybrid approximation inspirals for $\iota \gtrsim 90^{\circ}$ is probably not reliable, it is worth reexamining the behavior for $\iota \leqslant 90^{\circ}$. Good examples of this behavior are the plots for $\iota=80^{\circ}$ (lower leftmost panels of Figs. 3 and 4). In these cases, the cos $\iota$ terms in Eqs. (2.5) and (2.6) will be small but positive. Indeed, we see that the trajectories shown in this case are somewhat odd. Consider the trajectories that begin at $\left(p_{i}, e_{i}\right)=(20 M, 0.2)$. The points where the eccentricity evolution switches sign are at rather large semi-latus rectum ( $p \sim 16.5 M$ for $a=0.5 M ; p$ $\sim 25 \mathrm{M}$ for $a=0.9 \mathrm{M}$ ). This is quite a bit further out than is seen in any analysis of radiation reaction on equatorial orbits $[7,8,12]$. We suspect that this behavior is likewise an artifact of the weak-field fluxes, and do not trust the hybrid approximation's predictions for inspirals near $\iota=90^{\circ}$.

We conclude that the hybrid inspiral scheme - the weakfield fluxes (2.5) and (2.6) plus the "constant inclination" rule (3.9) applied to exact, strong-field Kerr geodesics - is, in most cases, reliable and accurate enough to be used for exploring issues in LISA's data analysis. In some cases, the hybrid scheme will not be accurate enough: the weak-field fluxes behave badly when the orbit goes too deep into the strong field, and so we do not trust this scheme's results when $r_{p} \leqslant 5 M$. Also, the spin correction terms in Eqs. (2.5) and (2.6) become either very small or very large relative to the leading quadrupole term when $\iota \sim 90^{\circ}$, and so we do not trust the hybrid approximation for inclination angles $80^{\circ} \lesssim \iota$ $\lesssim 120^{\circ}$. More rigorous strong-field analyses will be needed in order to validate the inspiral behavior at these inclination angles.

In all cases in which the inspiral behavior is reasonable, we find that small body's orbits will have significant eccentricity upon reaching the separatrix. Eccentricity will be a significant factor that must be incorporated into plans for LISA data analysis.

\section{CONJECTURE: APPROXIMATE $\dot{\boldsymbol{Q}}$ FOR GENERIC ZOOM-WHIRL ORBITS}

We have repeatedly emphasized that the hybrid approximation presented here is reliable as long as the orbiting body does not come too close to the central black hole. This excludes shallow inclination orbits of rapidly rotating black holes - an unfortunate exclusion, since those orbits are in many cases very strongly "stamped" by the features of the strong-field spacetime, and thus may be the most interesting inspiral sources observed by LISA [20]. These are also the orbits for which the "constant inclination $\dot{Q}$ " rule (3.9) is most likely to be inaccurate, since they are deepest in the 

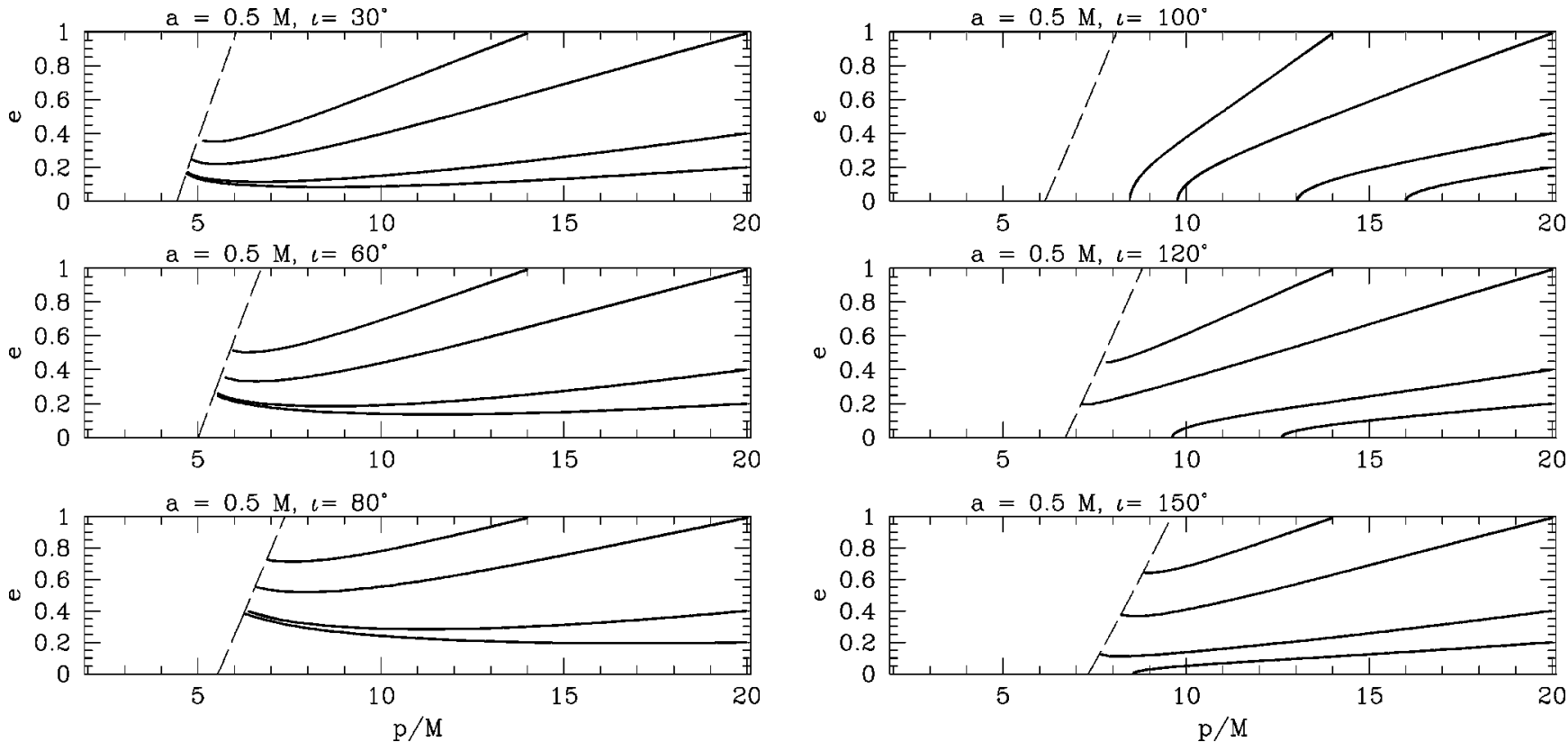

FIG. 3. Comparing generic inspiral at several inclination angles into a hole with spin $a=0.5 M$. In all plots the dashed line represents the separatrix between stable and unstable orbits; because we force the inspiral to lie in surfaces of constant $\iota$, there exists a separatrix in the $p$ - $e$ plane of each such surface. We use the hybrid inspiral scheme to evolve orbits with initial parameters $\left(p_{i}, e_{i}\right)=(20 M, 0.2),(20 M, 0.4)$, $(20 M, 0.99)$, and $(14 M, 0.99)$, and $\iota=30^{\circ}, 60^{\circ}, 80^{\circ}, 100^{\circ}, 120^{\circ}$, and $150^{\circ}$. Inspirals that are roughly "prograde" (have $\left.\iota<90^{\circ}\right)$ are qualitatively similar to the equatorial inspirals shown in Fig. 1: there is a turnaround in the eccentricity evolution, so that all inspirals finish with a substantial non-zero eccentricity. By contrast, the roughly "retrograde" inspirals $\left(\iota>90^{\circ}\right)$ exhibit rather different behavior: particularly when the inclination is not too far from $90^{\circ}$, many inspirals completely circularize, reaching $e=0$. As discussed in the text, we believe this behavior is incorrect.
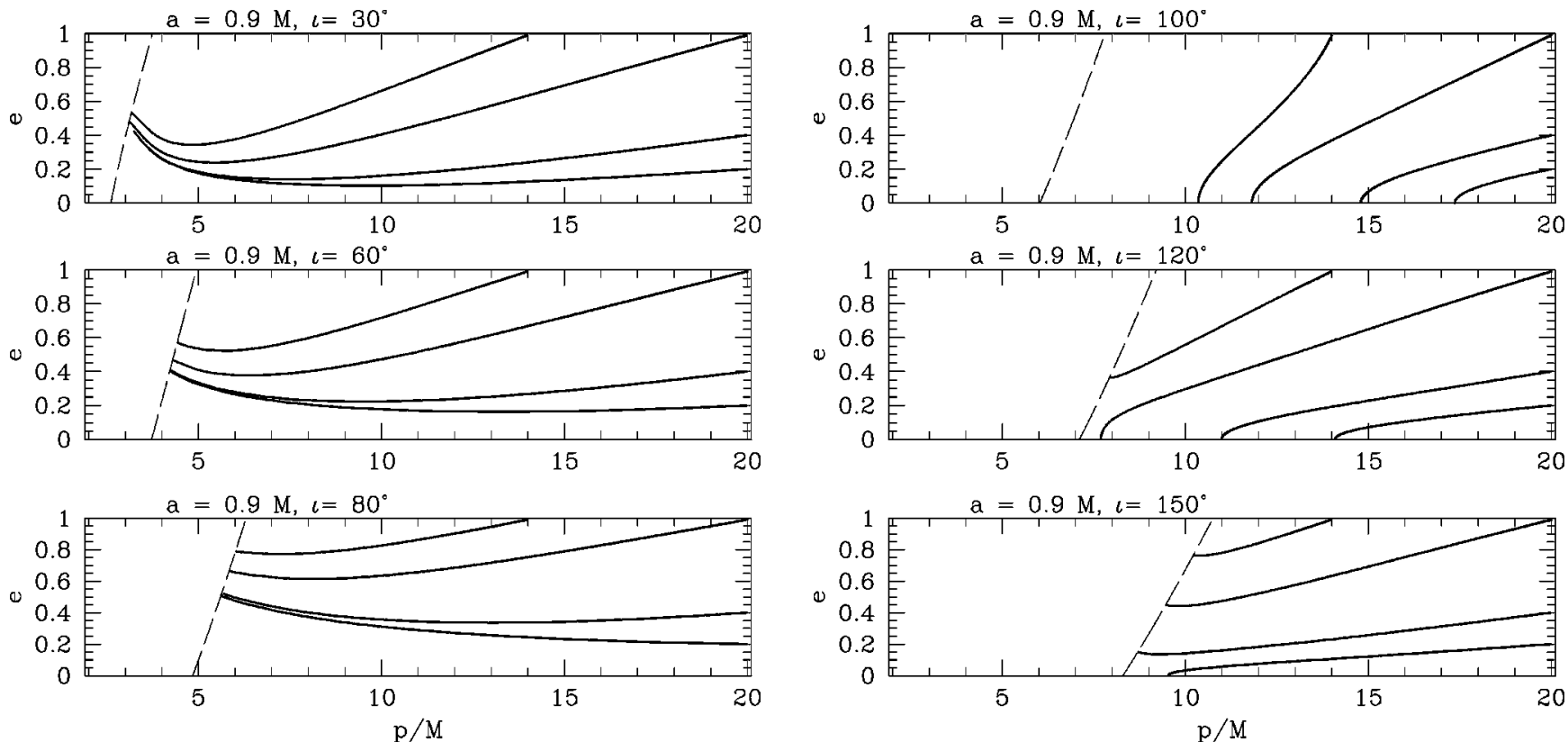

FIG. 4. Comparing generic inspiral at several inclination angles into a hole with spin $a=0.9 M$. Aside from the faster black hole spin, the trajectories shown here have identical initial parameters as those shown in Fig. 3. The inspirals for $\iota<90^{\circ}$ are again qualitatively similar to equatorial inspirals, shown in Fig. 2. In particular, we see that at shallow inclination angle, the growth in eccentricity is very large. We believe this is because these orbits go so deeply into the strong field that the weak-field flux formulas used in the hybrid approximation are no longer reliable. We also see the rapid circularization of inspirals when $\iota$ is greater than but near $90^{\circ}$, very similar to the behavior encountered for spin $a=0.5 \mathrm{M}$. 
Kerr black hole's strong field and are most likely to feel the asphericity of the Kerr spacetime. Ultimately, self-force computations will provide the toolsets needed to rigorously compute strong-field inspirals in this regime, and will sidestep all difficulties regarding the calculation of $\dot{Q}$. In the meantime, while such computations remain unavailable, it is worth exploring other possibilities that may provide accurate approximations to the Carter constant's evolution.

Strong-field eccentric equatorial orbits of rapidly rotating holes have a "zoom-whirl" character [12]: orbits near the separatrix in the $p$-e plane "whirl" around the black hole repeatedly near periastron, so that the amount of azimuth $\phi$ accumulated in a single radial cycle (apastron to periastron to apastron) is much greater than $2 \pi$. During this whirl phase, the body's motion is very close to a circular orbit. Exploratory studies [21] show that this behavior carries over to non-equatorial orbits, particularly for a fairly shallow inclination angle.

The equatorial zoom-whirl orbits studied in Ref. [12] radiate energy and angular momentum as if they were nearly circular:

$$
\dot{E} \approx \Omega_{\phi} \dot{L}_{z},
$$

where $\Omega_{\phi}$ is the frequency associated with azimuthal motion. This property follows quite naturally from the motion of a test body in a zoom-whirl orbit: a large fraction of the orbital period is spent "whirling" in the vicinity of the periastron, where the motion is nearly circular. This is also the part of the orbit where the body is closest to the black hole and thus where most of the radiation will be generated. Thus, the radiation from a zoom-whirl orbit should be very similar to radiation from a circular orbit, which is exactly what Eq. (5.1) suggests. Extrapolating this behavior to generic zoomwhirl orbits, we expect that most of the radiated $E, L_{z}$, and $Q$ will come from the motion of the body near a (generalized) separatrix in the $(p, \iota, e)$ phase space. The whirl motion of such orbits will be well described as nearly circular and inclined.

Following Kennefick and Ori [14], we can write the Carter constant as

$$
Q=G\left(r, E, L_{z}\right)-\Delta u_{r}^{2},
$$

where

$$
G=\Delta^{-1}\left[E\left(r^{2}+a^{2}\right)-a L_{z}\right]^{2}-\left(L_{z}-a E\right)^{2}-r^{2},
$$

with $\Delta=r^{2}-2 M r+a^{2}$ and $u_{r}$ denoting the radial component of the body's four-velocity. (The function that we have denoted $G$ is written $H$ in [14].) It is then straightforward to show that [14]

$$
\dot{Q}=G_{, E} \dot{E}+G_{, L_{z}} \dot{L}_{z}-\frac{2 \Sigma u^{r}}{u^{t}} F_{r},
$$

where $\Sigma=r^{2}+a^{2} \cos ^{2} \theta$ and $F_{r}$ is the radial component of the self-force. It is the unknown last term in this equation that presently prohibits the calculation of $\dot{Q}$ for generic orbits.
For strictly circular orbits, on the other hand, this term is absent since $u^{r}=0$. The remaining expression $\dot{Q}=\dot{Q}\left(\dot{E}, \dot{L}_{z}\right)$ allows the explicit calculation of $\dot{Q}$; this is how Hughes evolves circular, inclined orbits by reading the fluxes $\dot{E}$ and $\dot{L}_{z}$ at infinity and down the hole $[10,11]$.

For a zoom-whirl orbit and for motion near the periastron, $r \approx r_{p}$, so we should have $u^{r} \approx 0$; consequently, the unknown term in Eq. (5.4) should be negligible. Our conjecture is that the resulting expression for $\dot{Q}$ describes the evolution of the Carter constant for all generic zoom-whirl orbits and with increasing accuracy as the orbit approaches the separatrix. We emphasize that this approximation should hold even for orbits deep in the black hole's strong-field. This conjecture could become a practical tool once a code that calculates $\dot{E}$ and $\dot{L}_{z}$ for generic orbits is developed. Furthermore, a direct comparison between Eqs. (3.9) and (5.4) should be a useful guide for the accuracy of the $\iota=$ const rule in strong-field situations. Future computation of the self-force will provide the ultimate test for both approximations.

\section{CONCLUDING DISCUSSION}

The hybrid approximation presented in this paper should be a valuable tool for the gravitational-wave astrophysics community as studies of LISA's data analysis requirements begin, and thence models of the waves generated by compact bodies spiraling into massive black holes become needed. Such approximate "kludged" waveforms are obviously too crude to actually be used in future measurements of compact bodies spiraling into massive black holes; data analysis strategies based on waveforms built from rigorous strong-field radiation reaction will be needed. Waveforms from approximate inspiral models should be adequate to begin the process of developing a data analysis infrastructure. For example, they will be useful for counting the number of analysis filters needed, assessing the computational cost of data analysis, and experimenting with data analysis techniques. As rigorous and reliable waveform models become available, they can simply be dropped into the codes and infrastructure developed using the hybrid approximation.

Because this approximation combines the exact strongfield Kerr geodesics with weak-field radiation reaction formulas, it is somewhat limited: inspiral cannot go too deeply into the strong field, thereby making it inaccurate for shallow $\left(\iota \lesssim 20^{\circ}\right)$ inspirals of rapidly rotating $(a \geq 0.85 M)$ holes. Also, the $\cos \iota$ dependence of terms within the flux formulas behaves badly near $\iota \sim 90^{\circ}$, so that the approximation is probably not reliable within an inclination range $80^{\circ} \lesssim \iota$ $\lesssim 120^{\circ}$. Despite these limitations, we have found the hybrid approximation reliably and robustly duplicates many of the inspiral properties that we expect will be found when it is possible to study these systems using truly strong-field gravitational radiation reaction. In particular, it produces inspiral trajectories that retain substantial non-zero eccentricity just before plunging into the hole, as is expected from strongfield analyses in special cases $[7,8,12]$. We emphasize this point because the harmonic structure of gravitational waves from eccentric orbits is quite a bit different from that of 
waves generated by circular orbits. The residual eccentricity of typical inspirals is likely to impact data analysis rather strongly.

Obviously, waveforms constructed from hybrid approximation inspirals are by no means the ultimate models that will be needed for LISA data analysis - we strongly advocate continuing to develop techniques for understanding strong-field radiation reaction. Future insight from such studies may make it possible to improve the hybrid approximation. Even when strong-field radiation reaction is mature enough to model arbitrary compact body inspirals, the calculation may be computationally expensive, so that an approximation scheme of some sort may remain useful.

Although our overall goal is to provide a tool that can be used to model the gravitational waves produced by compact body inspiral, we have presented no such waves in this paper. That is the next step. The calculations we have presented explicitly construct the parameter space trajectories $\left[E(t), L_{z}(t), Q(t)\right]$ describing an inspiral. It is then a simple matter to combine such a trajectory with the geodesic equations for the Kerr spacetime [22] to produce the BoyerLindquist coordinate space inspiral $[r(t), \theta(t), \phi(t)]$. This set of functions specifies the world line of the inspiraling body, and one can use it to compute the gravitational waveform seen by distant observers (see, for example, Ref. [23]). Codes to perform this next step are under development [21]; results should be presented in the near future.

\section{ACKNOWLEDGMENTS}

We thank Kip Thorne for pressing us to develop "fast and dirty" techniques to compute inspiral waveforms, and Teviet Creighton for helping to test and debug the code that underlies parts of this analysis. K. G. thanks B. S. Sathyaprakash and Nils Andersson for useful interactions related to this work and also acknowledges support from PPARC Grant PPA/G/0/1999/0214. S.A.H. is supported by NSF Grant PHY-9907949. D.K. is partially supported by NSF Grant PHY-0099568.

\section{APPENDIX A}

The occurrence of a "third" orbital constant $Q$ in axisymmetric gravitational fields is not an exclusive feature of general relativity. For example, it is familiar from Newtonian celestial mechanics applied to orbital motion in galactic gravitational potentials (see, for example, Ref. [24], where the third constant is denoted $I$ ). The departure of $Q$ from $L_{x}^{2}+L_{y}^{2}$ can then be attributed to the asphericity of the potential. If such a potential does not deviate very much from sphericity, $L^{2}$ (the square of the total angular momentum) turns out to be almost constant, so that $Q$ should be, after all, nearly $L^{2}-L_{z}^{2}$.

It is straightforward to check whether this behavior of $L^{2}$ occurs in Kerr spacetime. The definition we use for $L^{2}$ is identical to that used in Schwarzschild spacetime,

$$
L^{2}=p_{\theta}^{2}+(\sin \theta)^{-2} p_{\phi}^{2},
$$
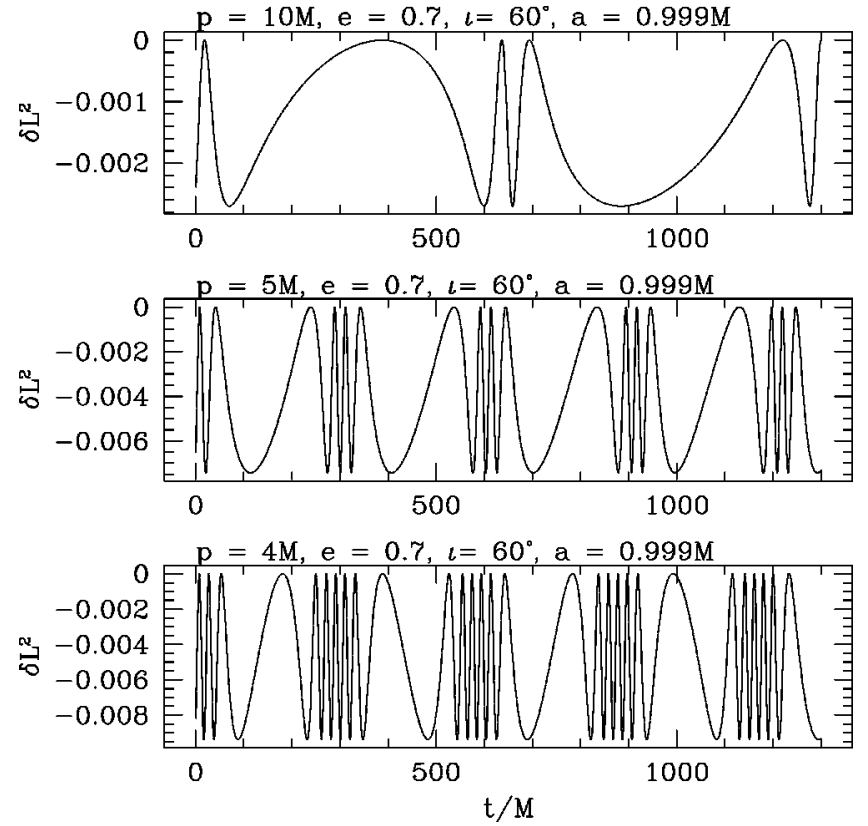

FIG. 5. Examining our notion of "total angular momentum" for strong-field Kerr black hole orbits. Each panel compares the angular momentum squared $L^{2} \equiv p_{\theta}^{2}+(\sin \theta)^{-2} p_{\phi}^{2}$ to $Q+L_{z}^{2}$ : the quantity plotted is $\delta L^{2} \equiv L^{2} /\left(Q+L_{z}^{2}\right)-1$. The top panel shows these quantities over an orbit with $p=10 M$, the center panel the quantities over an orbit with $p=5 M$, and the bottom over an orbit $p=4 M$. In all cases, the orbits have eccentricity $e=0.7$, inclination $\iota=60^{\circ}$, and are about a hole with spin $a=0.999 M$. Even deep in the strong field, $L^{2}$ differs very little from $Q+L_{z}^{2}$ - the greatest deviation in this sample is about $1 \%$. Since $Q+L_{z}^{2}$ is a constant by definition, this also shows that $L^{2}$ is approximately conserved over the orbit.

where $p_{a}$ denotes the particle's four-momentum. For the Carter constant $Q$ we have [22]

$$
Q=p_{\theta}^{2}+(\sin \theta)^{-2} p_{\phi}^{2}-p_{\phi}^{2}+a^{2} \cos ^{2} \theta\left(1-E^{2}\right) .
$$

Combining these two expressions gives

$$
Q=L^{2}-L_{z}^{2}+a^{2} \cos ^{2} \theta\left(1-E^{2}\right) .
$$

In other words, $Q$ can be interpreted as the projection of the total angular momentum on the equatorial plane, modulo the "aspherical" term $a^{2} \cos ^{2} \theta\left(1-E^{2}\right)$. This interpretation makes sense when the aspherical term is small - that is, when $a$ $\ll M$ (slow rotation) and/or $E \approx 1$ (weak-field orbits). In practice, we find that this term is often significantly smaller than the preceding terms even for motion in strong-field regions of rapidly rotating holes. We illustrate this in Fig. 5, showing how the quantity $\delta L^{2} \equiv L^{2} /\left(Q+L_{z}^{2}\right)-1$ varies with time for a variety of generic orbits around a rapidly spinning hole.

Examining Fig. 5, we see that $L^{2}$ deviates very little from $Q+L_{z}^{2}$ even when the small body is deep in the black hole's strong field - in this sample, the difference is no more than about $1 \%$. This shows that interpreting $Q$ as a squared projection of angular momentum into the equatorial plane is sensible. Because $Q+L_{z}^{2}$ is a constant quantity, this figure also demonstrates that $L^{2}$ is nearly constant. This is exactly 
what we expect for motion in an axisymmetric potential that is almost spherical. These pieces of evidence suggest that the Kerr spacetime is not as "aspherical" as we might have expected, at least for the purposes of this argument, lending credence to our suggestion that the " $\iota=$ const" assumption should be reliable, as discussed in the paper's main body.

\section{APPENDIX B}

This appendix contains explicit expressions (in terms of $E, L_{z}, Q$ and their derivatives) for the various functions appearing in the formulas (2.14) for the rates $\dot{p}, \dot{E}, i$. First,

$$
\begin{aligned}
H= & Q_{, p} E_{, e} L_{z, \iota}-Q_{, p} E_{, \iota} L_{z, e}-Q_{, e} E_{, p} L_{z, \iota} \\
& +Q_{, e} E_{, \iota} L_{z, p}+Q_{, \iota} E_{, p} L_{z, e}-Q_{, \iota} E_{, e} L_{z, p} .
\end{aligned}
$$

For $\dot{p}$ we find

$$
b_{p}=Q_{, \iota} L_{z, e}-Q_{, e} L_{z, \iota}
$$

$$
\begin{aligned}
& c_{p}=E_{, \iota} Q_{, e}-E_{, e} Q_{, \iota}, \\
& d_{p}=E_{, e} L_{z, \iota}-E_{, \iota} L_{z, e} .
\end{aligned}
$$

For $\dot{e}$ we find

$$
\begin{aligned}
& b_{e}=L_{z, \iota} Q_{, p}-Q_{, \iota} L_{z, p}, \\
& c_{e}=Q_{, \iota} E_{, p}-E_{, \iota} Q_{, p}, \\
& d_{e}=E_{, \iota} L_{z, p}-E_{, p} L_{z, \iota} .
\end{aligned}
$$

Finally, for $i$ the coefficients are

$$
\begin{gathered}
b_{\iota}=Q_{, e} L_{z, p}-Q_{, p} L_{z, e}, \\
c_{\iota}=Q_{, p} E_{, e}-Q_{, e} E_{, p}, \\
d_{\iota}=E_{, p} L_{z, e}-E_{, e} L_{z, p} .
\end{gathered}
$$

[1] K. Danzmann, et al., "LISA-Proposal for a LaserInterferometric Gravitational Wave Detector in Space," MaxPlanck-Institut für Quantenoptic, Report MPQ 177, 1993; K. Danzmann, et al., "LISA-Laser Interferometer Space Antenna, Pre-Phase A Report," Max-Planck-Institut für Quantenoptic, Report MPQ 233, 1998.

[2] M. Freitag, Class. Quantum Grav. 18, 4033 (2001); S. Sigurdsson and M.J. Rees, Mon. Not. R. Astron. Soc. 284, 318 (1997); S. Sigurdsson, Class. Quantum Grav. 14, 1425 (1997).

[3] F.D. Ryan, Phys. Rev. D 56, 1845 (1997); 56, 7732 (1997).

[4] S.A. Hughes, Class. Quantum Grav. 18, 4067 (2001).

[5] S. Chandrasekhar, The Mathematical Theory of Black Holes (Oxford University Press, New York, 1983).

[6] S.A. Teukolsky, Phys. Rev. Lett. 29, 1114 (1972); S.A. Teukolsky, Astrophys. J. 185, 635 (1973); M. Sasaki and T. Nakamura, Prog. Theor. Phys. 67, 1788 (1982).

[7] T. Apostolatos, D. Kennefick, A. Ori, and E. Poisson, Phys. Rev. D 47, 5376 (1993); D. Kennefick, ibid. 58, 064012 (1998).

[8] C. Cutler, D. Kennefick, and E. Poisson, Phys. Rev. D 50, 3816 (1994).

[9] Y. Mino, M. Sasaki, M. Shibata, H. Tagoshi, and T. Tanaka, Prog. Theor. Phys. Suppl. 128, 1 (1998).
[10] S.A. Hughes, Phys. Rev. D 61, 084004 (2000).

[11] S.A. Hughes, Phys. Rev. D 64, 064004 (2001).

[12] K. Glampedakis and D. Kennefick, Phys. Rev. D 66, 044002 (2002).

[13] F.D. Ryan, Phys. Rev. D 53, 3064 (1996).

[14] D. Kennefick and A. Ori, Phys. Rev. D 53, 4319 (1996).

[15] Y. Mino, Ph.D. thesis, Kyoto University, 1996.

[16] Y. Mino, M. Sasaki, and T. Tanaka, Phys. Rev. D 55, 3457 (1997); T.C. Quinn and R.M. Wald, ibid. 56, 3381 (1997); L.M. Burko, Phys. Rev. Lett. 84, 4529 (2000); H. Nakano, Y. Mino, and M. Sasaki, Prog. Theor. Phys. 106, 339 (2001); L. Barack and A. Ori, Phys. Rev. D 61, 061502 (2000); C. Lousto, Phys. Rev. Lett. 84, 5251 (2000).

[17] E.S. Phinney, astro-ph/0108028.

[18] P.C. Peters and J. Mathews, Phys. Rev. 131, 435 (1963).

[19] F.D. Ryan, Phys. Rev. D 52, 5707 (1995).

[20] S.A. Hughes, Phys. Rev. D 63, 064016 (2001).

[21] T.D. Creighton and S.A. Hughes (unpublished).

[22] C.W. Misner, K.S. Thorne, and J.A. Wheeler, Gravitation (Freeman, San Francisco, 1973), Chap. 33.

[23] W.H. Press, Phys. Rev. D 15, 965 (1977).

[24] J. Binney and S. Tremaine, Galactic Dynamics (Princeton University Press, Princeton, NJ, 1987), Chap. 3. 